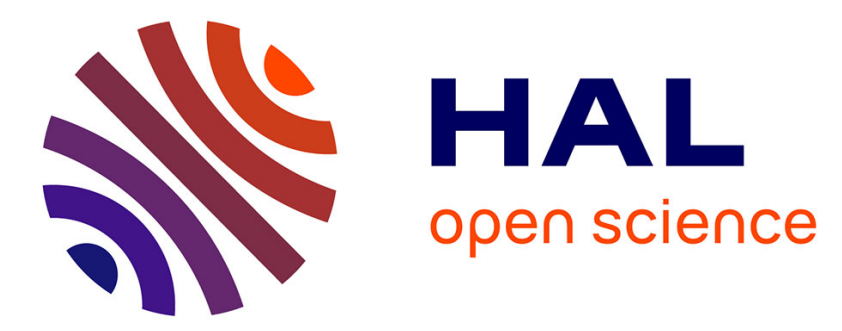

\title{
Space-time block coding for single-carrier block transmission DS-CDMA downlink
}

F. Petre, Geert Leus, Luc Deneire, M.G.E. Engels, M. Moonen, H. de Man

\section{To cite this version:}

F. Petre, Geert Leus, Luc Deneire, M.G.E. Engels, M. Moonen, et al.. Space-time block coding for single-carrier block transmission DS-CDMA downlink. IEEE Journal on Selected Areas in Communications, 2003, 21 (3), pp.350-361. 10.1109/JSAC.2003.809630 . hal-00187763

\section{HAL Id: hal-00187763 https://hal.science/hal-00187763}

Submitted on 15 Nov 2007

HAL is a multi-disciplinary open access archive for the deposit and dissemination of scientific research documents, whether they are published or not. The documents may come from teaching and research institutions in France or abroad, or from public or private research centers.
L'archive ouverte pluridisciplinaire HAL, est destinée au dépôt et à la diffusion de documents scientifiques de niveau recherche, publiés ou non, émanant des établissements d'enseignement et de recherche français ou étrangers, des laboratoires publics ou privés. 


\title{
Space-Time Block Coding for Single-Carrier Block Transmission DS-CDMA Downlink
}

\author{
Frederik Petré, Student Member, IEEE, Geert Leus, Member, IEEE, Luc Deneire, Member, IEEE, \\ Marc Engels, Member, IEEE, Marc Moonen, Member, IEEE, and Hugo De Man, Fellow, IEEE
}

\begin{abstract}
The combination of space-time block coding (STBC) and direct-sequence code-division multiple access (DS-CDMA) has the potential to increase the performance of multiple users in a cellular network. However, if not carefully designed, the resulting transmission scheme suffers from increased multiuser interference (MUI), which dramatically deteriorates the performance. To tackle this MUI problem in the downlink, we combine two specific DS-CDMA and STBC techniques, namely single-carrier block transmission (SCBT) DS-CDMA and time-reversal STBC. The resulting transmission scheme allows for deterministic maximum-likelihood (ML) user separation through low-complexity code-matched filtering, as well as deterministic ML transmit stream separation through linear processing. Moreover, it can achieve maximum diversity gains of $N_{T} N_{R}(L+1)$ for every user in the system, irrespective of the system load, where $N_{T}$ is the number of transmit antennas, $N_{R}$ the number of receive antennas, and $L$ the order of the underlying multipath channels. In addition, it turns out that a low-complexity linear receiver based on frequency-domain equalization comes close to extracting the full diversity in reduced, as well as full load settings. In this perspective, we also develop two (recursive) least squares methods for direct equalizer design. Simulation results demonstrate the outstanding performance of the proposed transceiver compared to competing alternatives.
\end{abstract}

Index Terms-Block transmission, direct-sequence code-division multiple access (DS-CDMA), equalizer design, frequency-selective fading channels, multiple-input-multiple-output (MIMO), space-time block coding (STBC).

\section{INTRODUCTION}

D IRECT-SEQUENCE code-division multiple access (DS-CDMA) has emerged as the predominant multiple access technique for third-generation $(3 \mathrm{G})$ cellular systems because it increases capacity and facilitates network planning in a cellular network. In the downlink, these systems rely on the orthogonality of the spreading codes to separate the different user signals. However, for increasing chip rates, the

Manuscript received May 1, 2002; revised November 1, 2002. The work of F. Petré was supported in part by the IWT. The work of G. Leus was supported in part by the FWO-Vlaanderen. This paper was presented in part at the EURASIP European Signal Processing Conference (EUSIPCO), Toulouse, France, September 3-6, 2002.

F. Petré, M. Engels, and H. De Man are with Interuniversity Micro-Electronics Center (IMEC), Leuven, Belgium and also with the Department of Electrical Engineering (ESAT), Katholieke Universiteit Leuven (KULeuven), Leuven, Belgium (e-mail: frederik.petre@imec.be; marc.engels@imec.be; hugo.deman@imec.be).

G. Leus and M. Moonen are with the Department of Electrical Engineering (ESAT), Katholieke Universiteit Leuven (KULeuven), Leuven, Belgium (e-mail: geert.leus@esat.kuleuven.ac.be; marc.moonen@esat.kuleuven.ac.be).

L. Deneire is with the I3S Laboratory, University of Nice, F-06903 Sophia-

Antipolis Cedex, France (e-mail: deneire@i3s.unice.fr).

Digital Object Identifier 10.1109/JSAC.2003.809630 time-dispersive nature of the multipath channel destroys the orthogonality amongst users, giving rise to multiuser interference (MUI). Chip-level equalization completely or partially restores the orthogonality and, hence, allows to suppress the MUI; see, e.g., [1] and [2]. However, this requires multiple receive antennas (two for chip rate sampling in a single-cell context) to guarantee a zero-forcing (ZF) solution for any set of well-conditioned channels [3]. On the other hand, single-carrier block transmission (SCBT) DS-CDMA, recently proposed as chip-interleaved block-spread (CIBS) CDMA in [4], only requires a single receive antenna because it effectively deals with the frequency-selectivity of the channel through zero padding (ZP) the chip blocks. Moreover, SCBT-DS-CDMA preserves the orthogonality amongst users regardless of the underlying multipath channel, which enables deterministic maximum-likelihood (ML) user separation through low-complexity code-matched filtering [4]. Increased equalization flexibility and reduced complexity are other benign properties that favor SCBT-DS-CDMA for broadband downlink transmission compared to conventional DS-CDMA [5]. However, the spectral efficiency and, hence, the user data rate of single-input-single-output (SISO) SCBT-DS-CDMA systems is limited by the received signal-to-noise ratio (SNR).

As opposed to SISO systems, multiple-input-multiple-output (MIMO) systems that employ $N_{T}$ transmit and $N_{R}$ receive antennas, have been shown to realize an $N_{\min }$-fold capacity increase in rich scattering environments [6]-[8], where $N_{\min }=\min \left\{N_{T}, N_{R}\right\}$ is called the multiplexing gain. Both spatial multiplexing and space-time coding (STC) are popular MIMO communication techniques that do not require any channel state information (CSI) at the transmitter. On the one hand, spatial multiplexing (SM), also known as BLAST, achieves high spectral efficiencies by transmitting independent data streams from the different transmit antennas [9] (see, also [10]). SM requires, however, at least as many receive as transmit antennas $\left(N_{R} \geq N_{T}\right)$, which seriously impairs cost-efficient implementations at the mobile station. On the other hand, STC achieves high quality-of-service (QoS) through diversity and coding gains by introducing both spatial and temporal correlations between the transmitted signals [11]-[13]. As opposed to SM, STC enables cheap implementations at the mobile station, since it supports any number of receive antennas. In this perspective, space-time block coding (STBC) techniques, introduced in [12] for $N_{T}=2$ transmit antennas, and later generalized in [13] for any number of transmit antennas, are particularly appealing because they facilitate ML detection with simple linear processing. However, these STBC techniques have been originally designed for frequency-flat 


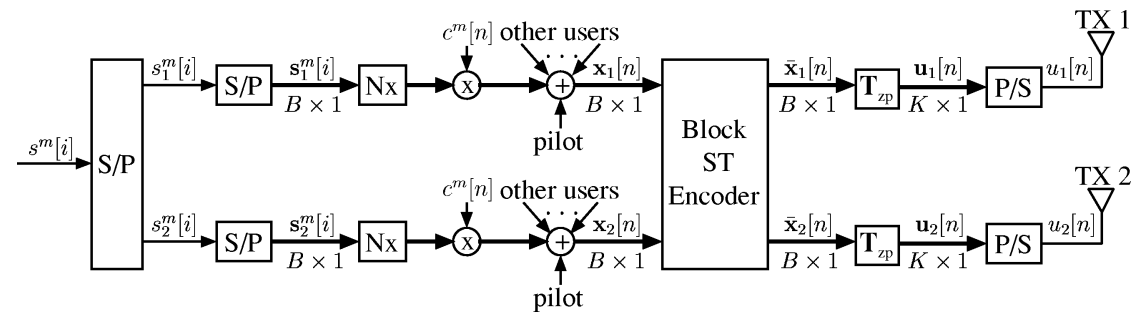

Fig. 1. Base station transmission scheme.

fading channels exploiting only multiantenna diversity of order $N_{T} N_{R}$. Therefore, time-reversal (TR) STBC techniques, originally proposed in [14] for single-carrier serial transmission, have been recently combined with SCBT in [15] and [16] for signalling over frequency-selective fading channels. While [15] only exploits multiantenna diversity, [16] exploits multiantenna as well as multipath diversity and achieves maximum diversity gains of order $N_{T} N_{R}(L+1)$ over frequency-selective fading channels, where $L$ is the order of the underlying multipath channels.

Until now, research on STBC techniques has mainly focused on point-to-point MIMO communication links, thereby neglecting the multiple-access technique in the design of the transmission scheme. Given the recent success of DS-CDMA, this clearly justifies the need for a transceiver that combines STBC with DS-CDMA. However, if not designed properly, the resulting communication scheme suffers from an $N_{T}$-fold increase of the MUI, which has a detrimental effect on the performance.

In this paper, we combine TR-STBC with SCBT-DS-CDMA for point-to-multipoint (downlink) frequency-selective fading MIMO communication links. The proposed transceiver preserves the orthogonality amongst users, as well as transmit streams regardless of the underlying multipath channels. This allows for deterministic ML user separation through low-complexity code-matched filtering, as well as deterministic ML transmit stream separation through linear processing. Moreover, applying ML Viterbi equalization for every transmit stream separately guarantees symbol recovery. Therefore, maximum diversity gains of $N_{T} N_{R}(L+1)$ can be achieved for every user in the system, irrespective of the system load. We will pursue, however, a slightly suboptimal approach that combines the transmit stream separation and equalization steps into one single linear equalization step. This enables the use of low-complexity equalizer design algorithms that circumvent the need for explicit channel estimation. In this perspective, we propose two [recursive (R)] least squares (LS) methods for direct equalizer design that act on a per-tone basis in the frequency-domain (FD). Both methods exploit the presence of a code-division multiplexed pilot (CDMP) (similar to the common pilot channel (CPICH) in $3 \mathrm{G}$ systems [17]) but differ in the amount of additional a priori information they assume to determine the equalizer coefficients. Moreover, they benefit from the ML user separability of the proposed transceiver.

Another alternative to remove MUI deterministically in a ST-coded multiuser setup has been recently reported in [18]. The transceiver of [18] combines generalized multicarrier (GMC) CDMA, originally developed in [19], with the STBC techniques of [13] but implemented on a per-carrier basis. However, similar to all MC systems, single-user GMC-CDMA transmissions generally suffer from high peak-to-average-power-ratio (PAPR), which requires expensive radio frequency (RF) front-ends and/or additional signal processing to compensate for the nonlinear effects introduced by the front-end. ${ }^{1}$ As opposed to [18], our transceiver is based on SCBT-DS-CDMA which allows for perfectly constant-modulus (CM) single-user transmissions, at least if we replace the zeros by known symbols [4], [20].

\section{Downlink Transceiver Design}

Let us consider the downlink of a ST block-coded SCBT-DS-CDMA system with $M$ active mobile stations. The base station is equipped with $N_{T}$ transmit (TX) antennas whereas the mobile station of interest is equipped with $N_{R}$ receive $(\mathrm{RX})$ antennas. In the following, we will first describe the base station transmitter design followed by the channel model and the mobile station receiver design.

\section{A. Transmitter Design}

The block diagram in Fig. 1 describes the ST block-coded downlink SCBT-DS-CDMA transmission scheme (where only the $m$ th user is explicitly shown), that transforms the $M$ user data symbol sequences $\left\{s^{m}[i]\right\}_{m=1}^{M}$ and the pilot symbol sequence $s^{p}[i]$ into $N_{T}$ ST block-coded multiuser chip sequences $\left\{u_{n_{t}}[n]\right\}_{n_{t}=1}^{N_{T}}$ with a rate $1 / T_{c}$. For conciseness, we limit ourselves to the case of $N_{T}=2$ transmit antennas and briefly discuss the more general case of $N_{T}>2$ transmit antennas at the end of Section II. Referring back to Fig. 1, the $m$ th user's data symbol sequence $s^{m}[i]$ (similarly for the pilot symbol sequence $s^{p}[i]$ ) is first demultiplexed into $N_{T}$ parallel lower rate sequences $\left\{s_{n_{t}}^{m}[i]:=s^{m}\left[i N_{T}+n_{t}-1\right]\right\}_{n_{t}=1}^{N_{T}}$. Unlike the traditional approach of symbol spreading that operates on a single symbol, we apply here block spreading that operates on a block of symbols; block spreading has also been used in, e.g., [4] and [21]. Specifically, each of the sequences $\left\{s_{n_{t}}^{m}[i]\right\}_{n_{t}=1}^{N_{T}}$ is serial-to-parallel converted into blocks of $B$ symbols, leading to the symbol block sequences $\left\{\mathbf{s}_{n_{t}}^{m}[i]:=\left[s_{n_{t}}^{m}[i B], \ldots, s_{n_{t}}^{m}[(i+1) B-1]\right]^{T}\right\}_{n_{t}=1}^{N_{T}}$ that are subsequently spread by a factor $N$ with the user composite code sequence $c^{m}[n]$ (pilot composite code sequence $c^{p}[n]$ ). For example, $c^{m}[n]$ is the multiplication of a short orthogonal Walsh-Hadamard spreading code that is mobile station specific

\footnotetext{
${ }^{1}$ There exist special cases of GMC-CDMA that have no PAPR problem, but they constitute a special SCBT-DS-CDMA design [19].
} 


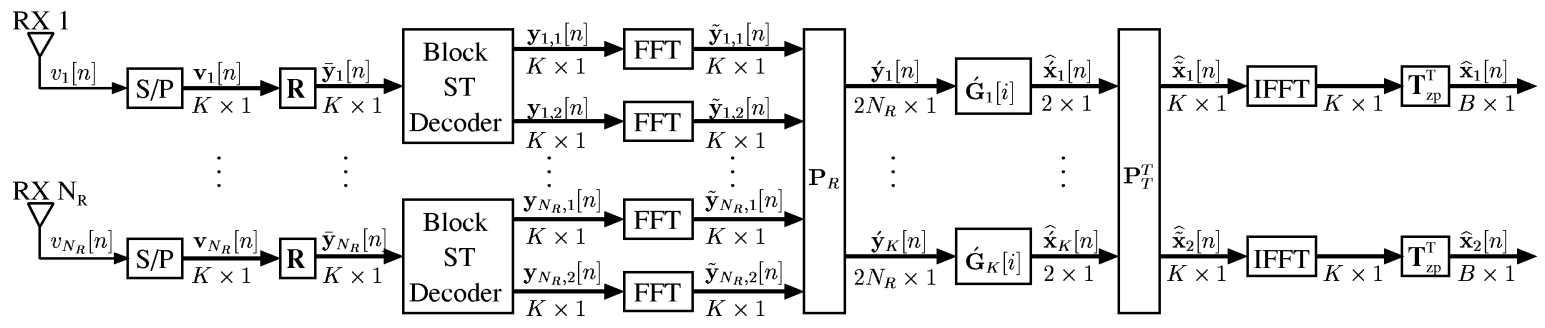

Fig. 2. Initial part of mobile station reception scheme.

(pilot specific) and a long overlay scrambling code that is base station specific. At this point, it is important to note that all $N_{T}$ parallel symbol block sequences are spread by the same composite code sequence. For each of the $N_{T}$ parallel streams, the different user chip block sequences and the pilot chip block sequence are added, resulting into the $n_{t}$ th multiuser chip block sequence

$$
\mathbf{x}_{n_{t}}[n]=\sum_{m=1}^{M} \mathbf{s}_{n_{t}}^{m}[i] c^{m}[n]+\mathbf{s}_{n_{t}}^{p}[i] c^{p}[n], \quad i=\left\lfloor\frac{n}{N}\right\rfloor .
$$

For future discussions, we find it convenient to also define the $m$ th user's total data symbol block sequence $\mathbf{s}^{m}[i]:=\left[\mathbf{s}_{1}^{m}[i]^{T}, \mathbf{s}_{2}^{m}[i]^{T}\right]^{T}$ (similarly for the total pilot symbol block sequence $\mathbf{s}^{p}[i]$ ) and the total multiuser chip block sequence $\mathbf{x}[n]:=\left[\mathbf{x}_{1}[n]^{T}, \mathbf{x}_{2}[n]^{T}\right]^{T}$.

Unlike the traditional approach of performing ST encoding at the symbol level (see, e.g., [12] and [13]), we perform ST encoding at the block level (see also Fig. 1); this was also done in, e.g., [15], [16], and [22]. Following the TR-STBC approach for single-user point-to-point SCBT [16], we apply the TR-STBC approach to SCBT-DS-CDMA. Our ST encoder operates in the time-domain (TD) at the chip block level rather than at the symbol block level and takes the two multiuser chip blocks $\left\{\mathbf{x}_{n_{t}}[n]\right\}_{n_{t}=1}^{2}$ to output the following $2 B \times 2$ matrix of ST coded multiuser chip blocks

$$
\left[\begin{array}{cc}
\overline{\mathbf{x}}_{1}[2 n] & \overline{\mathbf{x}}_{1}[2 n+1] \\
\overline{\mathbf{x}}_{2}[2 n] & \overline{\mathbf{x}}_{2}[2 n+1]
\end{array}\right]=\left[\begin{array}{cc}
\mathbf{x}_{1}[n] & -\mathbf{P}_{B}^{(0)} \cdot \mathbf{x}_{2}[n]^{*} \\
\mathbf{x}_{2}[n] & \mathbf{P}_{B}^{(0)} \cdot \mathbf{x}_{1}[n]^{*}
\end{array}\right]
$$

where $\mathbf{P}_{J}^{(j)}$ is a $J \times J$ permutation matrix implementing a TR followed by a cyclic shift over $j$ positions [16]. At each time interval $n$, the ST coded multiuser chip blocks $\overline{\mathbf{x}}_{1}[n]$ and $\overline{\mathbf{x}}_{2}[n]$ are forwarded to the first and the second transmit antenna, respectively. From (2), we can easily verify that the transmitted multiuser chip block at time instant $2 n+1$ from one antenna is the time-reversed conjugate of the transmitted multiuser chip block at time instant $2 n$ from the other antenna (with a possible sign change). For frequency-flat fading channels, symbol blocking is not necessary, so $B=1$ and $\mathbf{P}_{B}^{(0)}=1$, and the ST encoder of (2) reduces to the well-known Alamouti scheme [12]. However, for frequency-selective fading channels, the permutation matrix $\mathbf{P}_{B}^{(0)}$ is necessary to facilitate the exploitation of both multiantenna, as well as multipath diversity [16].

At each transmit antenna $n_{t} \in\{1,2\}$, the $K \times B$ zero padding transmit matrix $\mathbf{T}_{z p}:=\left[\mathbf{I}_{B}, \mathbf{0}_{B \times \mu}\right]^{T}$, with $K=B+\mu$, pads a zero postfix of length $\mu$ to $\overline{\mathbf{x}}_{n_{t}}[n]: \mathbf{u}_{n_{t}}[n]=\mathbf{T}_{z p} \cdot \overline{\mathbf{x}}_{n_{t}}[n]$, the

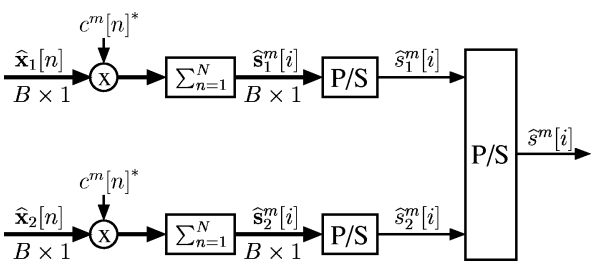

Fig. 3. Final part of mobile station reception scheme.

purpose of which will be clarified later. Finally, the resulting transmitted multiuser chip block sequence $\mathbf{u}_{n_{t}}[n]$ is parallel-to-serial converted into the corresponding scalar sequence $\left[u_{n_{t}}[n K], \ldots, u_{n_{t}}[(n+1) K-1]\right]^{T}:=\mathbf{u}_{n_{t}}[n]$.

\section{B. Channel Model}

The $N_{T}$ transmit antennas at the base station together with the $N_{R}$ receive antennas at the mobile station create a MIMO channel with $N_{T} N_{R}$ entries. Adopting a discrete-time baseband equivalent model, the chip-sampled received signal at the $n_{r}$ th receive antenna $v_{n_{r}}[n]$ is the superposition of a channel-distorded version of the signals from the $N_{T}$ transmit antennas, and can be written as

$$
v_{n_{r}}[n]=\sum_{n_{t}=1}^{N_{T}} \sum_{l=0}^{L_{n_{r}, n_{t}}} h_{n_{r}, n_{t}}[l] u_{n_{t}}[n-l]+w_{n_{r}}[n]
$$

where $h_{n_{r}, n_{t}}[l]$ is the chip-sampled finite-impulse response (FIR) channel from the $n_{t}$ th transmit antenna to the $n_{r}$ th receive antenna including the effect of transmit and receive filters, $L_{n_{r}, n_{t}}$ is the order of $h_{n_{r}, n_{t}}[l]$, and $w_{n_{r}}[n]$ denotes the additive Gaussian noise at the $n_{r}$ th receive antenna, which we assume to be additive white Gaussian noise (AWGN) with variance $\sigma_{w}^{2}$. Furthermore, we define $L$ as the upperbound on the channel orders: $L \geq \max _{n_{t}, n_{r}}\left\{L_{n_{r}, n_{t}}\right\} . L$ can be well approximated by $\left\lfloor\tau_{\max } / T_{c}\right\rfloor+1$, where $\tau_{\max }$ is the maximum delay spread within the given propagation environment [23].

\section{Receiver Design}

The block diagrams in Figs. 2 and 3 describe the reception scheme for the mobile station of interest (which we assume to be the $m$ th one), which transforms the different received sequences $\left\{v_{n_{r}}[n]\right\}_{n_{r}=1}^{N_{R}}$ into an estimate of the desired user's data symbol sequence $\hat{s}^{m}[i]$. Referring back to Fig. 2 and assuming perfect synchronization, at each receive antenna $n_{r} \in\left\{1, \ldots, N_{R}\right\}$, the received sequence $v_{n_{r}}[n]$ is serial-to-parallel converted into its corresponding block sequence $\mathbf{v}_{n_{r}}[n]:=\left[v_{n_{r}}[n K], \ldots, v_{n_{r}}[(n+1) K-1]\right]^{T}$. 
From the scalar input-output relationship in (3), we can derive the corresponding block input-output relationship as

$$
\begin{gathered}
\mathbf{v}_{n_{r}}[n]=\sum_{n_{t}=1}^{N_{T}}\left(\mathbf{H}_{n_{r}, n_{t}}[0] \cdot \mathbf{u}_{n_{t}}[n]+\mathbf{H}_{n_{r}, n_{t}}[1]\right. \\
\left.\cdot \mathbf{u}_{n_{t}}[n-1]\right)+\mathbf{w}_{n_{r}}[n]
\end{gathered}
$$

where $\mathbf{w}_{n_{r}}[n]:=\left[w_{n_{r}}[n K], \ldots, w_{n_{r}}[(n+1) K-1]\right]^{T}$ is the noise block sequence, $\mathbf{H}_{n_{r}, n_{t}}[0]$ is a $K \times K$ lower triangular Toeplitz matrix with entries $\left[\mathbf{H}_{n_{r}, n_{t}}[0]\right]_{p, q}=h_{n_{r}, n_{t}}[p-q]$, and $\mathbf{H}_{n_{r}, n_{t}}$ [1] is a $K \times K$ upper triangular Toeplitz matrix with entries $\left[\mathbf{H}_{n_{r}, n_{t}}[1]\right]_{p, q}=h_{n_{r}, n_{t}}[K+p-q]$ (see, e.g., [19] for a detailed derivation of the single-user SISO case). The time-dispersive nature of the multipath channel gives rise to so-called interblock interference (IBI) between successive blocks, which is modeled by the second term in (4). The $K \times K$ receive matrix $\mathbf{R}:=\mathbf{I}_{K}$ completely preserves each block of $\mathbf{v}_{n_{r}}[n]$ : $\overline{\mathbf{y}}_{n_{r}}[n]:=\mathbf{R} \cdot \mathbf{v}_{n_{r}}[n]=\mathbf{v}_{n_{r}}[n]$. Unlike classical OFDM systems, that eliminate the IBI by discarding the cyclic prefix at the receiver, here the IBI is entirely dealt with at the transmitter. Indeed, by choosing the length of the zero postfix to be at least the maximum channel order $\mu>L$, we obtain $\mathbf{R} \cdot \mathbf{H}_{n_{r}, n_{t}}[1] \cdot \mathbf{T}_{z p}=\mathbf{0}$, so the IBI is completely removed. Moreover, the zero postfix of the previous block $\mathbf{u}_{n_{t}}[n-1]$ acts as a cyclic prefix of the current block $\mathbf{u}_{n_{t}}[n]$, so that the Toeplitz matrix $\mathbf{H}_{n_{r}, n_{t}}[0]$ can be safely replaced by the $K \times K$ circulant matrix $\dot{\mathbf{H}}_{n_{r}, n_{t}}:=\mathbf{H}_{n_{r}, n_{t}}[0]+\mathbf{H}_{n_{r}, n_{t}}[1]$ through the identity $\mathbf{H}_{n_{r}, n_{t}}[0] \cdot \mathbf{T}_{z p}=\dot{\mathbf{H}}_{n_{r}, n_{t}} \cdot \mathbf{T}_{z p}$. In this way, we obtain a simplified block input-output relationship in the TD

$$
\overline{\mathbf{y}}_{n_{r}}[n]=\sum_{n_{t}=1}^{N_{T}} \dot{\mathbf{H}}_{n_{r}, n_{t}} \cdot \mathbf{T}_{z p} \cdot \overline{\mathbf{x}}_{n_{t}}[n]+\overline{\mathbf{z}}_{n_{r}}[n]
$$

where $\overline{\mathbf{z}}_{n_{r}}[n]:=\mathbf{R} \cdot \mathbf{w}_{n_{r}}[n]=\mathbf{w}_{n_{r}}[n]$ is the corresponding noise block sequence. Note that circulant matrices can be diagonalized by fast Fourier transform (FFT) operations [24], enabling simple per-tone frequency-domain (FD) equalization.

1) Space-Time Decoding: Following a similar approach as in [16] for point-to-point SCBT, ST decoding is performed in the TD at the chip block level rather than at the symbol block level. From the ST code design in (2), we can easily verify that

$$
\begin{aligned}
& \mathbf{T}_{z p} \cdot \overline{\mathbf{x}}_{1}[2 n+1]=-\mathbf{P}_{K}^{(B)} \cdot \mathbf{T}_{z p} \cdot \overline{\mathbf{x}}_{2}[2 n]^{*} \\
& \mathbf{T}_{z p} \cdot \overline{\mathbf{x}}_{2}[2 n+1]=\mathbf{P}_{K}^{(B)} \cdot \mathbf{T}_{z p} \cdot \overline{\mathbf{x}}_{1}[2 n]^{*}
\end{aligned}
$$

which can be interpreted as TR ST coding with permutation matrix $\mathbf{P}_{K}^{(B)}$ (rather than $\mathbf{P}_{B}^{(0)}$ ) applied to the zero-padded block sequences $\mathbf{T}_{z p} \cdot \mathbf{x}_{1}[n]^{*}$ and $\mathbf{T}_{z p} \cdot \mathbf{x}_{2}[n]^{*}$ (rather than to the block sequences $\mathbf{x}_{1}[n]^{*}$ and $\mathbf{x}_{2}[n]^{*}$ themselves). We can now consider two consecutive chip blocks $\overline{\mathbf{y}}_{n_{r}}[2 n]$ and $\overline{\mathbf{y}}_{n_{r}}[2 n+1]$ both satisfying the TD block input-output relationship of (5), and define $\mathbf{y}_{n_{r}, 1}[n]:=\overline{\mathbf{y}}_{n_{r}}[2 n]$ and $\mathbf{y}_{n_{r}, 2}[n]:=\mathbf{P}_{K}^{(B)} \cdot \overline{\mathbf{y}}_{n_{r}}[2 n+1]^{*}$ (see, also, Fig. 2). Aiming at low-complexity FD processing, we transform $\mathbf{y}_{n_{r}, 1}[n]$ and $\mathbf{y}_{n_{r}, 2}[n]$ into the FD by defining $\tilde{\mathbf{y}}_{n_{r}, 1}[n]:=\mathbf{F}_{K} \cdot \mathbf{y}_{n_{r}, 1}[n]$ and $\tilde{\mathbf{y}}_{n_{r}, 2}[n]:=\mathbf{F}_{K} \cdot \mathbf{y}_{n_{r}, 2}[n]$, where $\mathbf{F}_{K}$ is the $K \times K$ FFT matrix (see also Fig. 2). By exploiting the ST code structure of (6) and (7) like in [16], we arrive at the following FD block input-output relationship after ST decoding

$$
\begin{aligned}
\underbrace{\left[\begin{array}{c}
\tilde{\mathbf{y}}_{n_{r}, 1}[n] \\
\tilde{\mathbf{y}}_{n_{r}, 2}[n]
\end{array}\right]}_{\tilde{\mathbf{y}}_{n_{r}}[n]} & =\underbrace{\left[\begin{array}{cc}
\tilde{\mathbf{H}}_{n_{r}, 1} & \tilde{\mathbf{H}}_{n_{r}, 2} \\
\tilde{\mathbf{H}}_{n_{r}, 2}^{*} & -\tilde{\mathbf{H}}_{n_{r}, 1}^{*}
\end{array}\right]}_{\tilde{\mathbf{x}}[n]} \cdot \underbrace{\left[\begin{array}{l}
\left.\mathbf{F}_{K} \cdot \mathbf{T}_{z p} \cdot \mathbf{x}_{1}[n]\right] \\
\mathbf{F}_{K} \cdot \mathbf{T}_{z p} \cdot \mathbf{x}_{2}[n]
\end{array}\right]}_{\tilde{\mathbf{H}}_{n_{r}}} \\
& +\underbrace{\left[\begin{array}{c}
\tilde{\mathbf{z}}_{n_{r}, 1}[n] \\
\tilde{\mathbf{z}}_{n_{r}, 2}[n]
\end{array}\right]}_{\tilde{\mathbf{z}}_{n_{r}}[n]}
\end{aligned}
$$

where $\tilde{\mathbf{H}}_{n_{r}, n_{t}}:=\operatorname{diag}\left(\tilde{\mathbf{h}}_{n_{r}, n_{t}}\right)$ is a diagonal matrix, $\tilde{\mathbf{h}}_{n_{r}, n_{t}}$ is the FD channel response evaluated on the FFT grid, $\tilde{\mathbf{z}}_{n_{r}}[n]$ stacks the corresponding noise block sequences, and where $\overline{\mathbf{x}}_{1}[2 n]=\mathbf{x}_{1}[n]$ and $\overline{\mathbf{x}}_{2}[2 n]=\mathbf{x}_{2}[n]$ follow from (2).

From the structure of $\hat{\mathbf{H}}_{n_{r}}$ in (8), we can deduce that our transceiver preserves the orthogonality amongst transmit streams for each tone separately regardless of the underlying multipath channel. This allows for deterministic ML transmit stream separation through linear processing, assuming perfect CSI. A similar property was also encountered in the classical Alamouti scheme but only for point-to-point frequency-flat fading MIMO channels [12].

Stacking the contributions of the $N_{R} \mathrm{RX}$ antennas $\tilde{\mathbf{y}}[n]:=$ $\left[\tilde{\mathbf{y}}_{1}[n]^{T}, \ldots, \tilde{\mathbf{y}}_{N_{R}}[n]^{T}\right]^{T}$, finally leads to the following per-RXantenna FD data model:

$$
\tilde{\mathbf{y}}[n]=\tilde{\mathbf{H}} \cdot \tilde{\mathbf{x}}[n]+\tilde{\mathbf{z}}[n]
$$

where $\tilde{\mathbf{H}}:=\left[\tilde{\mathbf{H}}_{1}^{T}, \ldots, \tilde{\mathbf{H}}_{N_{R}}^{T}\right]^{T}$ is the per-RX-antenna channel matrix, and $\tilde{\mathbf{z}}[n]:=\left[\tilde{\mathbf{z}}_{1}[n]^{T}, \ldots, \tilde{\mathbf{z}}_{N_{R}}[n]^{T}\right]^{T}$ is the corresponding noise block sequence.

2) FD Equalization: Although one could argue that a maximum diversity transmission scheme, like the one we propose here, is only useful when an ML receiver is applied, we will show in the following that it is also useful when a suboptimal receiver is applied. Unlike the approach followed by [25], that applies ML linear transmit stream separation followed by ML Viterbi equalization, we combine these operations into one single linear equalization step, at the expense of some loss in ML optimality. As will be shown in Section III, this suboptimal approach enables the use of low-complexity per-tone equalizer design algorithms that circumvent the need for explicit channel estimation.

Defining the per-tone input block $\mathbf{x}[n]$ and the per-tone output block $\mathbf{y}[n]$ as

$$
\begin{aligned}
& \dot{\mathbf{x}}[n]:=\mathbf{P}_{T} \cdot \tilde{\mathbf{x}}[n]=\left[\dot{\mathbf{x}}_{1}[n], \ldots, \dot{\mathbf{x}}_{K}[n]\right]^{T} \\
& \dot{\mathbf{y}}[n]:=\mathbf{P}_{R} \cdot \tilde{\mathbf{y}}[n]=\left[\dot{\mathbf{y}}_{1}[n], \ldots, \dot{\mathbf{y}}_{K}[n]\right]^{T}
\end{aligned}
$$

where $\mathbf{P}_{T}$ permutes a per-TX-antenna ordering into a per-tone ordering and where $\mathbf{P}_{R}$ permutes a per-RX-antenna ordering into a per-tone ordering, we obtain the following per-tone data model:

$$
\dot{\mathbf{y}}[n]=\mathbf{H} \cdot \mathbf{x}[n]+\mathbf{z}[n]
$$

where $\mathbf{z}[n]:=\mathbf{P}_{R} \cdot \tilde{\mathbf{z}}[n]$ is the per-tone noise block. The per-tone channel matrix $\mathbf{H}$ is a block diagonal matrix, given by

$$
\hat{\mathbf{H}}:=\mathbf{P}_{R} \cdot \tilde{\mathbf{H}} \cdot \mathbf{P}_{T}^{T}=\operatorname{diag}\left\{\dot{\mathbf{H}}_{1}, \ldots, \dot{\mathbf{H}}_{K}\right\} .
$$


Looking at (8)-(11), it is important to note that each of the $2 N_{R} \times 2$ submatrices $\mathbf{H}_{k}$, with $k \in\{1, \ldots, K\}$, retains the orthogonality amongst transmit streams on a particular tone $k$, similarly to the Alamouti scheme for frequency-flat fading channels [12].

Armed with the model of (12), we can now discuss different equalization options that recover the multiuser chip block sequence $\mathbf{x}[n]$. Since ML Viterbi equalization, as used in [25], requires exponential complexity in the channel order $L$, we will only focus on low-complexity linear alternatives. A first possiblity is to apply a ZF chip equalizer [26]

$$
\dot{\mathbf{G}}_{\mathrm{ZF}}=\left(\mathbf{H}^{H} \cdot \mathbf{H}\right)^{-1} \cdot \mathbf{H}^{H}
$$

which completely eliminates the interchip interference (ICI) at the expense of excessive noise enhancement. A second possiblity is to apply a minimum mean-square error (MMSE) chip equalizer [26], that explicitly takes into account the noise and balances ICI eliminination with noise enhancement. The MMSE chip equalizer is expressed as

$$
\dot{\mathbf{G}}_{\mathrm{MMSE}}=\left(\mathbf{H}^{H} \cdot \mathbf{H}+\sigma_{w}^{2} \cdot \mathbf{R}_{\hat{x}}^{-1}\right)^{-1} \cdot \mathbf{H}^{H}
$$

where $\mathbf{R}_{\dot{x}}:=E\left\{\dot{\mathbf{x}}[n] \cdot \dot{\mathbf{x}}^{H}[n]\right\}$ is the input covariance matrix, and $\sigma_{w}^{2}$ is the AWGN variance. From (14) and (15), it is also clear that $\dot{G}_{\text {MMSE }}$ reduces to $\mathbf{G}_{\mathrm{ZF}}$ at high SNR.

It is important to note that the block diagonal per-tone structure of the channel matrix $\mathbf{H}$ in (13) is also reflected in both the ZF equalizer matrix $\mathbf{G}_{\mathrm{ZF}}$, as well as the MMSE equalizer matrix $\mathbf{G}_{\mathrm{MMSE}}$. So, both (14) and (15) actually capture $K$ parallel and independent equalizers: $\mathbf{G}:=\operatorname{diag}\left\{\mathbf{G}_{1}, \ldots, \mathbf{G}_{K}\right\}$, where $\mathbf{G}_{k}$ is a size $2 \times 2 N_{R}$ matrix that recovers the two parallel streams on the $k$ th tone only, with $k \in\{1, \ldots, K\}$ (see also Fig. 2).

3) User-Specific Detection: Stacking $N$ consecutive chip blocks $\dot{\mathbf{y}}[n]$ into $\mathbf{Y}[i]:=[\hat{\mathbf{y}}[i N], \ldots, \dot{\mathbf{y}}[(i+1) N-1]]$, we obtain the symbol block level equivalent of (12)

$$
\hat{\mathbf{Y}}[i]=\mathbf{H} \cdot \mathbf{X}[i]+\mathbf{Z}[i]
$$

where $\mathbf{X}[i]$ and $\mathbf{Z}[i]$ are similarly defined as $\mathbf{Y}[i]$. From (8) and (10), we also have

$$
\dot{\mathbf{X}}[i]=\mathbf{P}_{T} \cdot \mathcal{F}_{K} \cdot \mathcal{T}_{z p} \cdot \mathbf{X}[i]
$$

where $\mathbf{X}[i]:=[\mathbf{x}[i N], \ldots, \mathbf{x}[(i+1) N-1]]$ stacks $N$ consecutive chip blocks $\mathbf{x}[n], \mathcal{F}_{K}:=\operatorname{diag}\left\{\mathbf{F}_{K}, \mathbf{F}_{K}\right\}$ is the compound FFT matrix, and $\mathcal{T}_{z p}:=\operatorname{diag}\left\{\mathbf{T}_{z p}, \mathbf{T}_{z p}\right\}$ is the compound zero padding transmit matrix. From (1), it is also clear that

$$
\mathbf{X}[i]=\mathbf{S}^{d}[i] \cdot \mathbf{C}^{d}[i]^{T}+\mathbf{s}^{p}[i] \cdot \mathbf{c}^{p}[i]^{T}
$$

where the multiuser data symbol matrix $\mathbf{S}^{d}[i]:=\left[\mathbf{s}^{1}[i], \ldots\right.$, $\left.\mathbf{s}^{M}[i]\right]$ stacks the total data symbol blocks of the different active users at the $i$ th symbol block instant. The active multiuser code matrix $\mathbf{C}^{d}[i]:=\left[\mathbf{c}^{1}[i], \ldots, \mathbf{c}^{M}[i]\right]$ stacks the composite code vectors of the different active users at the $i$ th symbol block instant, where $\mathbf{c}^{m}[i]:=\left[c^{m}[i N], \ldots, c^{m}[(i+1) N-1]\right]^{T}$ is the $m$ th user's composite code vector used to spread its total data symbol block $\mathbf{s}^{m}[i]$. The total pilot symbol block $\mathbf{s}^{p}[i]$ and the pilot composite code vector $\mathbf{c}^{p}[i]$ are similarly defined as $\mathbf{s}^{m}[i]$ and $\mathbf{c}^{m}[i]$, respectively. For future discussions, we find it convenient to also define the inactive multiuser code matrix $\overline{\mathbf{C}}^{d}[i]:=$ $\left[\overline{\mathbf{c}}^{1}[i], \ldots, \overline{\mathbf{c}}^{\bar{M}}[i]\right]$ that stacks the $\bar{M}:=N-M-1$ unused composite code vectors $\overline{\mathbf{c}}^{\bar{m}}[i]:=\mathbf{c}^{M+\bar{m}}[i]$, with $\bar{m} \in\{1, \ldots, \bar{M}\}$. It is important to note that $\mathbf{c}^{p}[i], \mathbf{C}^{d}[i]$, and $\overline{\mathbf{C}}^{d}[i]$ together form an orthonormal basis of the $N$-dimensional code space.

Starting from (16), the obtained chip equalizer matrix $\mathbf{G}$, whether ZF or MMSE, may subsequently be used to extract the desired user's total data symbol block

$$
\hat{\mathbf{s}}^{m}[i]=\mathcal{T}_{z p}^{T} \cdot \mathcal{F}_{K}^{H} \cdot \mathbf{P}_{T}^{T} \cdot \mathbf{G} \cdot \mathbf{Y}[i] \cdot \mathbf{c}^{m}[i]^{*}
$$

where the estimate of the FD multiuser chip block matrix $\widehat{\tilde{\mathbf{X}}}[i]:=\mathbf{P}_{T}^{T} \cdot \mathbf{G}^{\prime} \cdot \hat{\mathbf{Y}}[i]$ is transformed to the TD by the compound inverse fast Fourier transform (IFFT) matrix $\mathcal{F}_{K}^{H}$ and has its zero postfix removed by the transpose of the compound ZP transmit matrix $\mathcal{T}_{z p}$. The resulting estimate of the TD multiuser chip block matrix $\widehat{\mathbf{X}}[i]:=\mathcal{T}_{z p}^{T} \cdot \mathcal{F}_{K}^{H} \cdot \widehat{\hat{\mathbf{X}}}[i]$ is finally descrambled and despread with the desired user's composite code vector $\mathbf{c}^{m}[i]$ to obtain an estimate of the desired user's total data symbol block $\hat{\mathbf{s}}^{m}[i]$. These operations are alternatively represented by Fig. 2 (see right-hand side) and Fig. 3 .

In the above procedure, we have first applied equalization at the chip block level followed by despreading with the desired user's composite code vector. However, we might interpret (19) differently by reversing the order of these operations, so first performing the despreading followed by equalization at the symbol block level. From (17) and (18), we can easily derive that

$$
\dot{\mathbf{X}}[i]=\dot{\mathbf{S}}^{d}[i] \cdot \mathbf{C}^{d}[i]^{T}+\dot{\mathbf{S}}^{p}[i] \cdot \mathbf{c}^{p}[i]^{T}
$$

where $\mathbf{S}^{d}[i]:=\mathbf{P}_{T} \cdot \mathcal{F}_{K} \cdot \mathcal{T}_{z p} \cdot \mathbf{S}^{d}[i]$ is the permuted version of the multiuser data symbol matrix $\mathbf{S}^{d}[i]$ after ZP and FFT processing, and likewise $\mathbf{s}^{p}[i]:=\mathbf{P}_{T} \cdot \mathcal{F}_{K} \cdot \mathcal{T}_{z p} \cdot \mathbf{s}^{p}[i]$ for the total pilot symbol block $\mathbf{S}^{p}[i]$. So, despreading (16) with the desired user's composite code vector $\mathbf{c}^{m}[i]$ leads to the following single-user data model

$$
\hat{\mathbf{Y}}[i] \cdot \mathbf{c}^{m}[i]^{*}=\mathbf{H} \cdot \dot{\mathbf{s}}^{m}[i]+\mathbf{Z}[i] \cdot \mathbf{c}^{m}[i]^{*}
$$

because of the orthonormality between the user and the pilot composite code vectors at each symbol instant. From (21), we can also conclude that our transceiver preserves the orthogonality amongst users regardless of the underlying multipath channels and succesfully converts (through despreading) a multiuser chip block equalization problem into a single-user symbol block equalization problem. Similarly to (14) and (15), we can then obtain ZF and MMSE symbol block equalizers that act on a per-tone basis in the FD. Note that a classical DS-CDMA transmission scheme does not possess this nice property.

Throughout Section II, we have limited our discussion to the case of $N_{T}=2$ transmit antennas and any number of receive antennas $N_{R}$. However, our transceiver design can be easily extended to the general case of $N_{T}>2$ transmit antennas by resorting to the generalized complex orthogonal designs (GCOD) of [13]. The difference is that the GCOD should be defined at the chip block level rather than at the scalar symbol level. In general, a GCOD of size $N_{T} B \times N_{D}$ depends on $N_{S}$ different multiuser chip blocks, where $N_{S}$ is the number of independent transmit streams, $N_{D}$ is the time span (measured in number of 
chip block instants), and $R:=N_{S} / N_{D}$ is the rate of the ST code. Previously, we implicitly assumed that $N_{S}=N_{T}=$ $N_{D}=2$, resulting in an Alamouti-like scheme with $R=1$. For $N_{T}>2, R=1 / 2 \mathrm{ST}$ code designs for SCBTs have been derived in [25]. In the end, we obtain the same per-tone data model of (12). Hence, the ZF and MMSE equalizers that we have developed in Section II-C2, as well as the equalizer design methods of Section III are directly applicable here as well. So, both (14) and (15) still capture $K$ parallel and independent equalizers: $\mathbf{\mathbf { G }}:=\operatorname{diag}\left\{\mathbf{G}_{1}, \ldots, \dot{\mathbf{G}}_{K}\right\}$, where $\mathbf{G}_{k}$ is now a size $N_{S} \times N_{R} N_{D}$ matrix that recovers the $N_{S}$ parallel streams on the $k$ th tone only.

\section{PRACTICAl EQualizer Design}

Until now, we have assumed perfect CSI at the receiver to calculate either ZF [see (14)] or MMSE [see (15)] type of equalizers. However, in practice the receiver needs to acquire and/or update CSI before the actual equalization and detection can take place. In the following, we derive two equalizer design methods, that directly determine the equalizer coefficients without having to estimate the channel first. LS methods for burst processing as well as recursive least squares (RLS) methods for adaptive processing are discussed.

Starting from (16) and assuming the channel matrix $\mathbf{H}$ to have full column rank and the input matrix $\mathbf{X}[i]$ to have full row rank, the matrix $\mathbf{G}^{\prime}$, for which $\mathbf{G}^{\prime} \cdot \mathbf{Y}[i]-\mathbf{X}[i]=\mathbf{0}$ in the absence of noise, is the $\mathrm{ZF}$ equalizer matrix given by (14). In the presence of noise, we have to solve the corresponding LS minimization problem, which we denote for convenience as

$$
\dot{\mathbf{G}}_{\mathrm{LS}}=\arg \min _{\mathbf{G}} \sum_{i=0}^{I-1}\|\dot{\mathbf{G}} \cdot \mathbf{Y}[i]-\dot{\mathbf{X}}[i]\|^{2}
$$

where we consider $I$ consecutive symbol block instants $i \in\{0, \ldots, I-1\}$, corresponding to a burst length of $N_{T} B I$ data symbols per user. Two distinct ways exist to solve this minimization problem. The first way constrains the equalizer matrix $\mathbf{G}$ to be fixed over the entire burst length (under the assumption that the channel matrix $\mathbf{H}$ remains constant as well) and leads to an LS type of burst processing algorithm. The second way updates the chip equalizer matrix Ǵ $[i]$ from one symbol block instant to the next and leads to a RLS type of adaptive processing algorithm. Note that a numerically stable square root information (SRI) type of RLS algorithm can be easily derived from its corresponding LS algorithm. Such an algorithm consists of a triangular QR decomposition (QRD) updating step and a triangular back substitution step [27].

Substituting (20) into (22), leads to (23), shown at the bottom of the page, which is an LS problem in both the per-tone equalizer matrix $\mathbf{G}$ and the per-tone multiuser total data symbol matrix $\dot{\mathbf{S}}^{d}[i]$. Taking (23) as a starting point, we will develop in the following two equalizer design methods, that differ in the amount of a priori information they exploit to determine the equalizer coefficients. The first method, coined CDMP-trained, only exploits the presence of a CDMP. The second method, coined semi-blind, additionally exploits knowledge of the unused composite code vectors in any practical CDMA system.

\section{A. CDMP-Trained Equalizer}

The CDMP-trained equalizer is calculated from the per-tone output matrices $\left\{\hat{\mathbf{Y}}_{i}[i]\right\}_{i=0}^{I-1}$ based on the knowledge of the pilot composite code vectors $\left\{\mathbf{c}^{p}[i]\right\}_{i=0}^{I-1}$ and the per-tone total pilot symbol blocks $\left\{\mathbf{s}^{p}[i]\right\}_{i=0}^{I-1}$. By despreading (23) with the pilot composite code vector $\mathbf{c}^{p}[i]$ and by relying on the orthonormality of the user and the pilot composite code vectors at each symbol instant, we obtain

$$
\dot{\mathbf{G}}_{\mathrm{LS}}=\arg \min _{\dot{\mathbf{G}}} \sum_{i=0}^{I-1}\left\|\dot{\mathbf{G}}^{\prime} \mathbf{\mathbf { Y }}[i] \cdot \mathbf{c}^{p}[i]^{*}-\mathbf{s}^{p}[i]\right\|^{2}
$$

which can be interpreted as follows. The equalized per-tone output matrix $\mathbf{G} \cdot \mathbf{Y}[i]$ is first despread with the pilot composite code vector $\mathbf{c}^{p}[i]$. The resulting equalized per-tone output matrix after despreading $\mathbf{G} \cdot \mathbf{Y}[i] \cdot \mathbf{c}^{p}[i]^{*}$ should then be as close as possible in a LS sense to the per-tone total pilot symbol block $\mathbf{S}^{p}[i]$.

\section{B. Semi-Blind Equalizer}

Compared to the CDMP-trained equalizer, the semi-blind equalizer additionally exploits knowledge of the inactive multiuser code matrices $\left\{\overline{\mathbf{C}}^{d}[i]\right\}_{i=0}^{I-1}$. By despreading (23) with the pilot composite code vector $\mathbf{c}^{p}[i]$ and the inactive multiuser code matrix $\overline{\mathbf{C}}^{d}[i]$, respectively, we obtain (25), shown at the bottom of the page, where $\alpha$ is a weighting factor. ${ }^{2}$ The LS problem of (25) consists of two different parts: a training-based part and a fully blind part. On the one hand, the training-based part, described by the first term of (25), corresponds to the CDMP-trained equalizer of (24). On the other hand, the fully blind part, described by the second term of (25), can be

${ }^{2}$ Note that for $\alpha=1(25)$ is equivalent to (23).

$$
\mathbf{G}_{L S}=\arg \min _{\mathfrak{G}} \sum_{i=0}^{I-1}\left\|\mathbf{G} \cdot \mathbf{Y}[i]-\mathbf{S}^{d}[i] \cdot \mathbf{C}^{d}[i]^{T}-\dot{\mathbf{S}}^{p}[i] \cdot \mathbf{c}^{p}[i]^{T}\right\|^{2}
$$

$$
\dot{\mathbf{G}}_{L S}=\arg \min _{\dot{\mathbf{G}}} \sum_{i=0}^{I-1}\left\{\left\|\dot{\mathbf{G}} \cdot \mathbf{Y}[i] \cdot \mathbf{c}^{p}[i]^{*}-\dot{\mathbf{s}}^{p}[i]\right\|^{2}+\alpha\left\|\dot{\mathbf{G}} \cdot \mathbf{Y}[i] \cdot \overline{\mathbf{C}}^{d}[i]^{*}\right\|^{2}\right\}
$$


interpreted as follows. The equalized per-tone output matrix $\mathbf{G} \cdot \mathbf{Y}[i]$ is first despread with the inactive multiuser code matrix $\overline{\mathbf{C}}^{d}[i]$. The resulting equalized per-tone output matrix after despreading $\mathbf{\mathbf { G }} \cdot \mathbf{Y}[i] \cdot \overline{\mathbf{C}}^{d}[i]^{*}$ should then be as small as possible in a LS sense, which actually corresponds to a minimum output energy (MOE) criterion. In addition, it suggests that the inactive composite code vectors can be interpreted as virtual pilot composite code vectors that continuously carry zero virtual pilot symbols.

The previous discussion justifies why this equalizer design method can be classified as a semi-blind method. The weighting factor $\alpha$ controls the importance of the fully blind part relative to the training-based part. Giving equal importance $(\alpha=1)$ to both the training-based and the fully blind part does not necessarily lead to the best performance. Similar observations were made for semi-blind channel estimation algorithms in [28] and [29]. For $\alpha=0$, the semi-blind equalizer reduces to the CDMPtrained equalizer of (24). For $\alpha \rightarrow \infty$, the semi-blind equalizer converges to the purely blind equalizer. Clearly, an optimal $\alpha_{\text {opt }}$ should exist that leads to the best performance. However, a detailed analysis of $\alpha_{\mathrm{opt}}$ is beyond the scope of this paper. As will be demonstrated in Section IV, like in [29], $\alpha=0.1$ gives close to optimal performance. It is also worth noting that for a fully loaded system, so when $M=N-1$, all composite code vectors are in use and the fully blind term of (25) becomes obsolete. This means that for a fully loaded system the semi-blind approach reduces to the regular CDMP-trained approach.

Similar CDMP-trained, semi-blind and purely blind methods for traditional DS-CDMA were studied in [2], [3], and [30]-[32]. However, here the block despreading operations $\mathbf{Y}[i] \cdot \mathbf{c}^{p}[i]^{*}$ and $\mathbf{Y}[i] \cdot \overline{\mathbf{C}}^{d}[i]^{*}$ deterministically remove the MUI caused by any other signals and succesfully convert a chip block equalization problem into an equivalent symbol block equalization problem [see also (21)].

\section{Complexity Comparison}

With $T:=N_{R} N_{D}$ the number of equalizer taps per tone and per transmit stream (see the end of Section II), we evaluate the operation count of the two RLS adaptive equalizers, in terms of multiply/accumulates. A multiply/accumulation is an operation of the type $d=c+a . b$ that involves one complex multiplication, one complex addition, three complex reads and one complex write. Hence, the number of corresponding data transfers is four times the number of multiply/accumulates. Also, we only account for the updating phase, where the new values of the equalizer coefficients are determined. The detection phase, where these equalizer coefficients are used to detect the desired user's symbol block, is common to both adaptive equalizers. Both phases are executed continuously at a rate $R_{s} /\left(N_{S} B\right)$, where $R_{s}$ is the symbol rate.

According to (24), the RLS CDMP-trained updating consists of three computational steps per time update, namely first a pilot despreading step, then a triangular QRD-updating step, and finally a triangular backsubstitution step. On the one hand, the pilot despreading step only requires $\mathcal{O}(K N T)$ operations. On the other hand, both the triangular QRD-updating and the triangular backsubstitution step require $\mathcal{O}\left(K N_{S} T^{2}\right)$ operations. Overall, the RLS CDMP-trained updating requires
$K N_{S} 3 T^{2}+K\left(4 N_{S}+N\right) T$ operations, involving a complexity of $\mathcal{O}\left(K N_{S} T^{2}\right)$. It is important to note however that these operations need to be executed at a rate which is $B N_{S}$ times smaller than the symbol rate $R_{s}$. According to (25), the virtual pilot despreading step and the triangular QRD-updating step of the RLS semi-blind updating are $(\bar{M}+1)$ times more complex than their respective counterparts in the RLS pilot-trained updating. Overall, the RLS semi-blind updating requires $K N_{S}(2 \bar{M}+3) T^{2}+K\left[\left(N+2 N_{S}\right) \bar{M}+\left(4 N_{S}+N\right)\right] T$ operations, involving a complexity of $\mathcal{O}\left(K N_{S} \bar{M} T^{2}\right)$. For specific values, we refer to the end of the next section.

\section{Simulation Results}

We consider the downlink of a ST block-coded SCBT-DS-CDMA system with, unless otherwise stated, $N_{T}=2$ transmit antennas at the base station and $N_{R}=2$ receive antennas at the mobile station of interest. Each user's quaternary phase-shift keying (QPSK) modulated data symbol sequence is demultiplexed into $N_{S}=2$ parallel lower rate sequences. Choosing the initial block length $B=12$, these sequences are block spread by a real orthogonal Walsh-Hadamard spreading code of length $N=16$ along with a complex random scrambling code. The ST encoder spans a time interval of $N_{D}=2$ chip block instants, resulting in a rate $R=N_{S} / N_{D}=1$. Furthermore, we assume that each channel is FIR with, unless otherwise stated, order $L=3$ and Rayleigh distributed channel taps of equal variance $1 /\left(N_{T} N_{R}(L+1)\right)$. In this way, the average received power is normalized with respect to the number of transmit, as well as the number of receive antennas. To satisfy the postfix condition $\mu \geq L$, we choose its length $\mu=4$, and correspondingly the transmitted block length $K=B+\mu=16$. The ST block-coded multiuser chip sequences are transmitted at the chip rate $R_{c}=3.84 \mathrm{MHz}$, resulting in a symbol rate $R_{s}=(1 / N) R(B / K) R_{c}=180 \mathrm{kHz}$. In the following, we simulate the average bit-error rate (BER) versus the average received SNR over 500 Monte Carlo channel realizations for three different test cases.

\section{A. Comparison With the Optimal ML Diversity Bound}

We test the proposed transceiver, employing a cascade of TR-STBC and SCBT-DS-CDMA at the transmitter and applying MMSE FD equalization based on perfect CSI at the receiver, for three different MIMO system setups $\left(N_{T}, N_{R}\right)$ : the $(1,1)$ setup, the $(2,1)$ setup with TX diversity only, and the $(2,2)$ setup with both TX and RX diversity. The system is fully loaded supporting $M=16$ active users. No pilot is present. The performance of each setup is compared with its corresponding optimal ML performance bound for $N_{T} N_{R}(L+1)$-fold diversity over Rayleigh-fading channels [23].

Fig. 4 depicts the results for frequency-selective channels with channel order $L=1$. Fixing the BER at $10^{-3}$ and focusing on the proposed transceiver, the $(2,1)$ setup outperforms the $(1,1)$ setup by $6 \mathrm{~dB}$. The $(2,2)$ setup achieves on its turn a $3.5-\mathrm{dB}$ gain compared to the $(2,1)$ setup. Comparing the simulated performance of the proposed transceiver with its corresponding ML diversity bound, it incurs a 4- $\mathrm{dB}$ loss for the $(1,1)$ setup, but only a $0.4-\mathrm{dB}$ loss for the $(2,2)$ setup. So, 


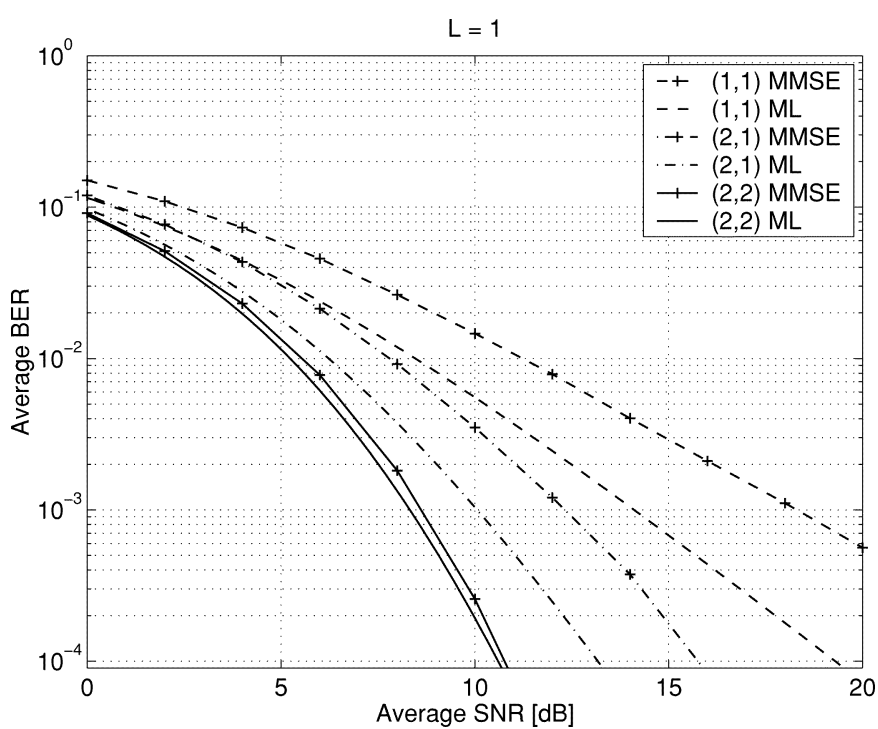

Fig. 4. Multiuser performance for channel order $L=1$.

the larger the number of TX and/or RX antennas, the better the proposed transceiver with linear receiver processing succeeds in extracting the full diversity of order $N_{T} N_{R}(L+1)$.

Fig. 5 shows the same results but now for frequency-selective channels with channel order $L=3$. Again fixing the BER at $10^{-3}$ and focusing on the proposed transceiver, the $(2,1)$ setup outperforms the $(1,1)$ setup by $4 \mathrm{~dB}$, whereas the $(2,2)$ setup achieves on its turn a $2-\mathrm{dB}$ gain compared to the $(2,1)$ setup. So, compared to Fig. 4, the corresponding gains are now smaller because of the inherently larger underlying multipath diversity.

\section{B. Comparison With Other ST-Coded CDMA Transceivers}

In the following, we compare four different ST-coded CDMA transceivers. The first transceiver (T1) applies the ST-coded DS-CDMA transmission scheme that was proposed for the UMTS and the IS-2000 WCDMA standards, also known as space-time spreading (STS) [33]. At the receiver, a space-time RAKE is applied similar to the time-only RAKE discussed in [33], but instead of using a time-only maximum ratio combiner (MRC) based on exact CSI, we use a space-time MRC based on exact CSI. The bandwidth efficiency of the first transceiver supporting $M_{1}$ users can be calculated as $\epsilon_{1}=M_{1} / N$, where $N$ is the length of the Walsh-Hadamard spreading codes.

The second transceiver (T2) employs a cascade of the classical downlink DS-CDMA transmission scheme and TR-STBC [34]. At the receiver, a time-domain ST chip equalizer is applied similar to the ones discussed in [34], but instead of using a chip equalizer that is trained with the pilot, we use an ideal MMSE chip equalizer based on perfect CSI. The bandwidth efficiency of the second transceiver supporting $M_{2}$ users can be determined as $\epsilon_{2}=M_{2} B /(B N+\mu)$, where $B$ is the initial block length, and $\mu$ is the length of the zero postfix.

The third transceiver (T3) is the one proposed in Section II that combines the SCBT-DS-CDMA transmission scheme with TR-STBC. At the receiver, a frequency-domain MMSE equalizer based on perfect CSI is used. The bandwidth efficiency of our transceiver supporting $M_{3}$ users can be calculated as $\epsilon_{3}=M_{3} B /(N(B+\mu))$.

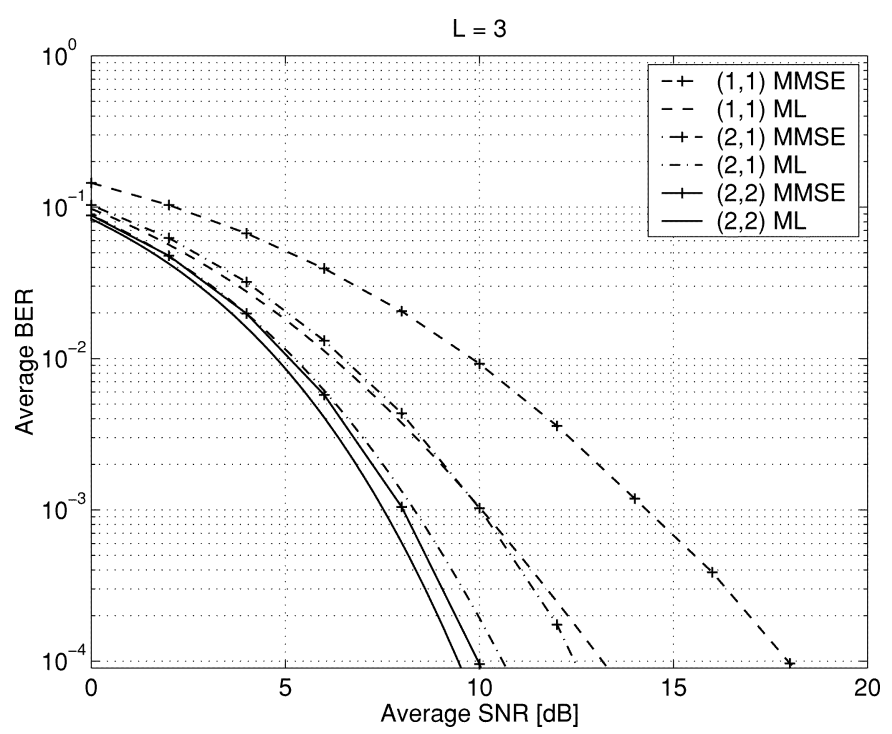

Fig. 5. Multiuser performance for channel order $L=3$.

The fourth transceiver (T4) combines the classical multicarrier (MC) DS-CDMA transmission scheme with the STBC techniques of [13], but implemented on a per-tone basis [35], [36]. At the receiver, the ST block decoding as well as the MMSE equalization are performed in the frequency-domain based on exact CSI. The bandwidth efficiency of the fourth transceiver supporting $M_{4}$ users can be determined as $\epsilon_{4}=M_{4} Q /(N(Q+\mu))$, where $Q$ is the number of tones, and $\mu$ is the length of the cyclic prefix.

In order to make a fair comparison between the four transceivers, we should force their bandwidth efficiencies to be the same $\epsilon_{1}=\epsilon_{2}=\epsilon_{3}=\epsilon_{4}$. This leads to the following relationship between the number of users to be supported by the different transceivers

$M_{2}=\frac{B N+\mu}{B N} M_{1}, \quad M_{3}=\frac{B+\mu}{B} M_{1}, \quad M_{4}=\frac{Q+\mu}{Q} M_{1}$.

With $B=12, Q=B, N=16$ and $\mu=4$, we can derive that $M_{2}=49 / 48 M_{1} \approx M_{1}$ and $M_{4}=M_{3}=4 / 3 M_{1}$. It is important to remark that for this comparison no pilot is present.

Fig. 6 compares the performance of the four transceivers for a small system load with $M_{1}=M_{2}=3$ and $M_{3}=M_{4}=4$. Also shown in the figure is the optimal ML performance bound for $N_{T} N_{R}(L+1)=16$-fold diversity over Rayleigh fading channels [23]. T2 and T3 have similar performance with a small advantage for T3 over T2 at high SNR. They both come close to extracting the full diversity, so both multiantenna as well as multipath diversity. As opposed to T2 and T3, T1 does not succeed in extracting the full diversity: at a BER of $10^{-4}$ it incurs a 3.8-dB loss compared to T3. Likewise, T4 does not succeed in extracting the full diversity either since it only exploits multiantenna diversity: at a BER of $10^{-4}$ it incurs a 3.4-dB loss compared to $\mathrm{T} 3$.

Fig. 7 depicts the same curves but now for a large system load with $M_{1}=M_{2}=12$ and $M_{3}=M_{4}=16$. Since both T3 and T4 are MUI-free ST-coded CDMA transceivers, their performance remains unaffected by the MUI. So, even at large 


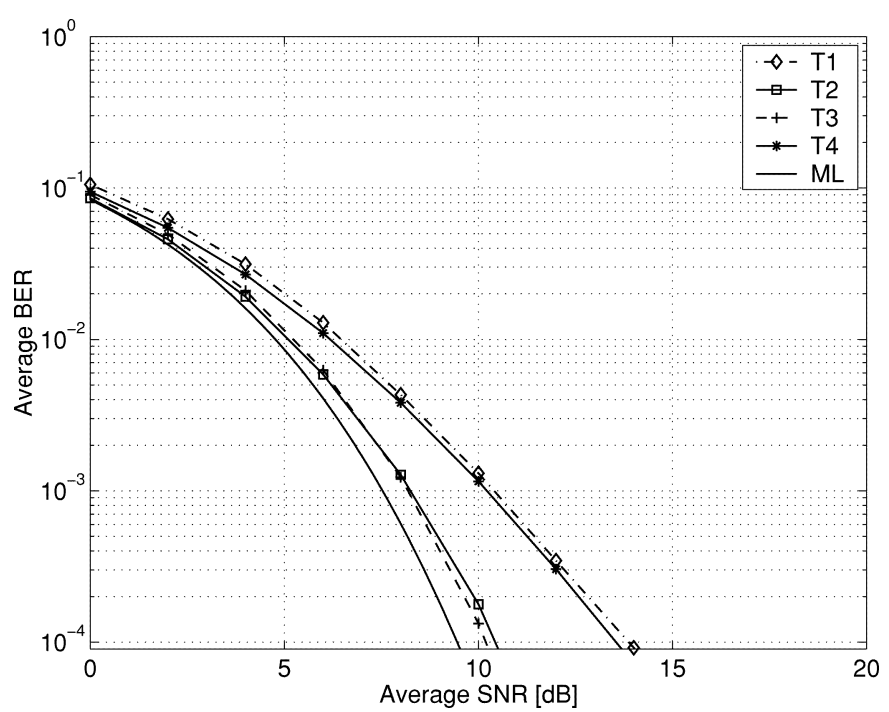

Fig. 6. Comparison for small system load.

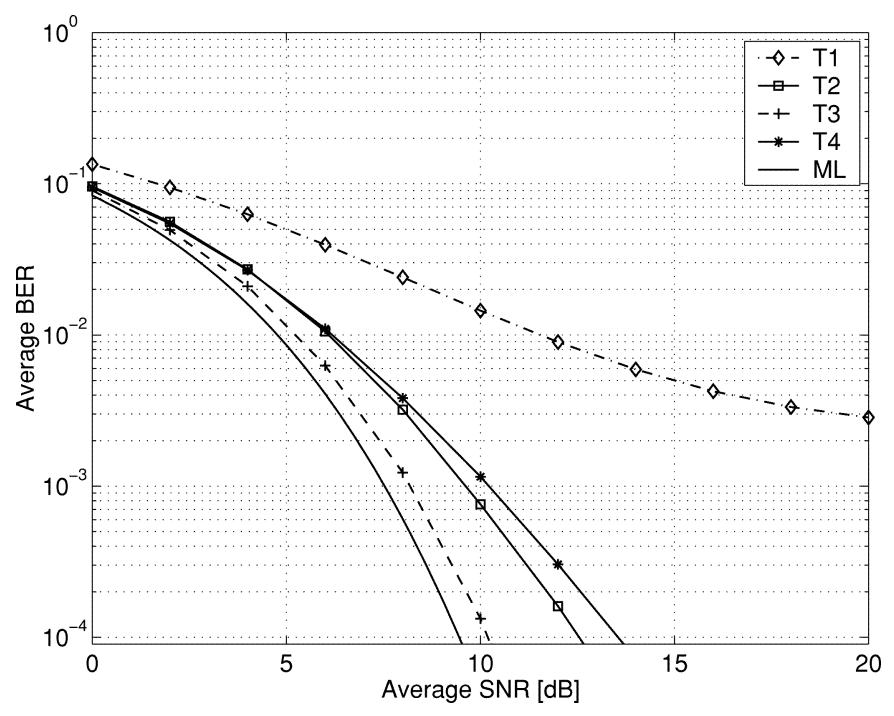

Fig. 7. Comparison for large system load.

system load, T3 comes close to extracting the full diversity: e.g., at a BER of $10^{-4}$ it only incurs a 0.6-dB loss compared to the optimal ML diversity bound. Unlike T3, T4 only exploits multiantenna diversity and incurs a 4-dB loss compared to the optimal ML diversity bound. As opposed to T3, T2 does not deterministically remove the MUI (T2 completely suppresses the MUI only at infinite SNR) and, therefore, fails in extracting the full diversity: e.g., at a BER of $10^{-4}$, T3 achieves a $2.4-\mathrm{dB}$ gain compared to $\mathrm{T} 2$. We also observe that $\mathrm{T} 1$ now performs poorly compared to T2, T3, and T4: e.g., at a BER of $3 \cdot 10^{-3}$, T3 achieves a 12.3-dB gain compared to $\mathrm{T} 1$. In contrast with $\mathrm{T} 3$ and T4 that deterministically remove the MUI, and with $\mathrm{T} 2$ that completely suppresses the MUI at infinite SNR, T1 does not completely suppress the MUI at high SNR. Hence, T1 suffers from a BER saturation level that increases with the system load $M_{1}$.

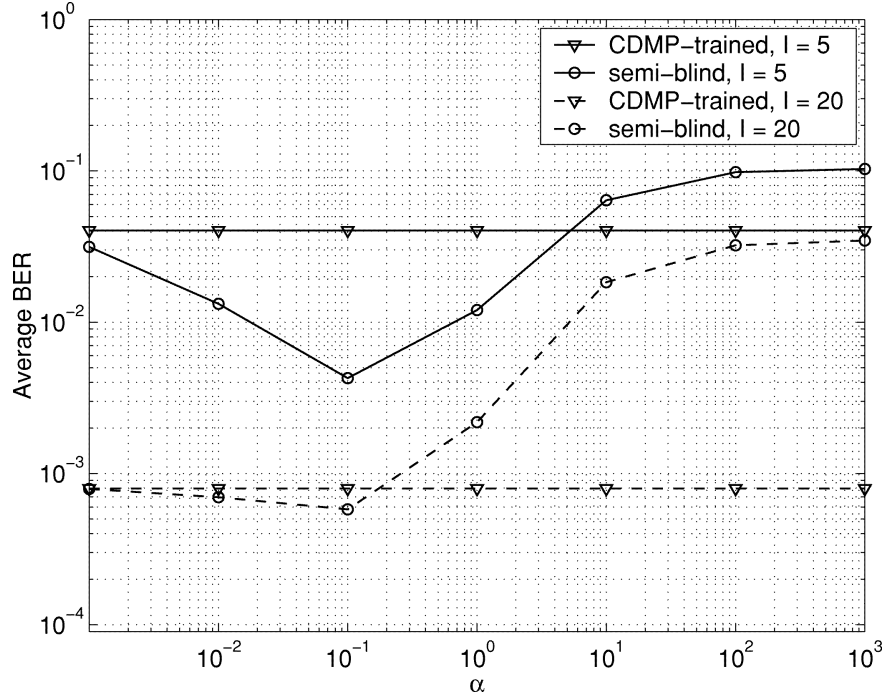

Fig. 8. Influence of $\alpha$ for $I=20$ and $I=5$ blocks, respectively.

\section{Comparison of the Different Equalizer Design Methods}

In order to test the equalizer design methods discussed in Section III, we employ the same system parameters as before (see the beginning of Section IV) with $M=7$ active users and one CDMP, similar to the so-called CPICH [17] in forthcoming $3 \mathrm{G}$ systems. Hence, the number of unused codes is $\bar{M}=$ $N-M-1=8$. As we already indicated in Section III-B, the performance of our semi-blind equalizer is quite sensitive to the choice of the weighting factor $\alpha$. Fixing the SNR to $10 \mathrm{~dB}$, Fig. 8 shows the influence of $\alpha$ when $I=20$ and $I=5$ symbol blocks are collected, respectively, to perform the LS equalizer estimators. For $\alpha \rightarrow 0$, the semi-blind equalizer converges to the CDMP-trained equalizer. For $\alpha \rightarrow \infty$, the semi-blind equalizer converges to the purely blind equalizer, that always performs worse than the CDMP-trained equalizer. These simulation results also indicate that $\alpha=0.1$ gives close to optimal performance. Moreover, the optimum is more pronounced when only a small number of symbol blocks is collected. Hence, we employ $\alpha=0.1$ for the following test cases.

Fig. 9 shows the BER versus SNR results when $I=20$ and $I=5$ symbol blocks are collected, respectively, to perform the LS equalizer design methods. Collecting $I=20$ symbol blocks and fixing the BER at $10^{-3}$, the performance of the semi-blind equalizer comes within $0.75 \mathrm{~dB}$ of the ideal MMSE equalizer with perfect CSI. Moreover, the semi-blind equalizer performs $0.4 \mathrm{~dB}$ better than the CDMP-trained equalizer. Considering only $I=5$ symbol blocks, the semi-blind equalizer outperforms the CDMP-trained equalizer by $7 \mathrm{~dB}$. The faster convergence stems from the fact that the semi-blind equalizer additionally exploits knowledge of the unused spreading codes, whereas the CDMP-trained equalizer only exploits the presence of a CDMP.

With $T=N_{R} N_{D}=4$ the number of equalizer taps per tone and per transmit stream, the CDMP-trained and the semi-blind adaptive equalizers are updated at a rate $R_{s} / N_{S} B=7.5 \mathrm{kHz}$. On the one hand, the CDMP-trained adaptive equalizer requires $23 \mathrm{Mops} / \mathrm{s}$ and a data transfer bandwidth of $92 \mathrm{Mwords} / \mathrm{s}$. On the other hand, the semi-blind adaptive equalizer requires $161 \mathrm{Mops} / \mathrm{s}$ and a data transfer bandwidth of $644 \mathrm{Mwords} / \mathrm{s}$. 


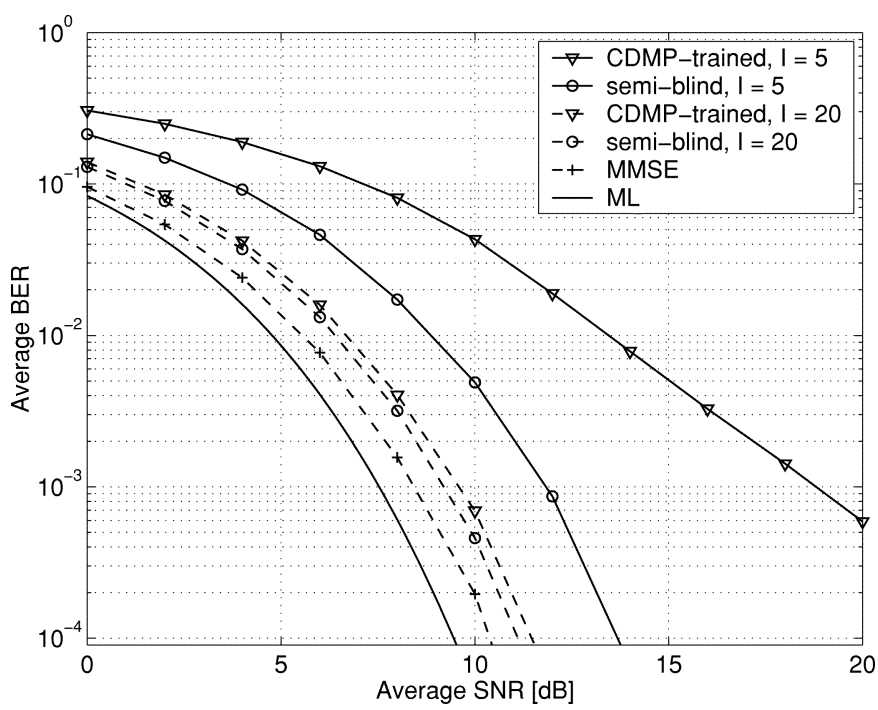

Fig. 9. Equalizer design with $I=20$ and $I=5$ blocks, respectively.

Hence, the semi-blind method involves a seven times higher complexity than the CDMP-trained method.

\section{CONCLUSION}

To enable significant performance improvements compared to $3 \mathrm{G}$ cellular systems, we have designed a novel ST blockcoded CDMA transceiver, that can yield gains of up to $12 \mathrm{~dB}$ in full load situations. To this end, we have combined two specific DS-CDMA and STBC techniques, namely SCBT-DS-CDMA on the one hand and TR-STBC on the other hand. The resulting transmission scheme allows for deterministic ML user separation through low-complexity code-matched filtering, as well as deterministic ML transmit stream separation through linear processing. Moreover, it can achieve maximum diversity gains of $N_{T} N_{R}(L+1)$ for every user in the system, irrespective of the system load. In addition, a low-complexity linear receiver based on frequency-domain equalization approaches the optimal ML performance (within $0.6 \mathrm{~dB}$ for a $(2,2)$ system) and comes close to extracting the full diversity in reduced as well as full load settings. In this perspective, we have also proposed two (recursive) LS based methods for direct equalizer design, coined CDMPtrained and semi-blind, respectively. Both methods exploit the presence of a CDMP and benefit from the ML user separability through code-matched filtering. When collecting a large number of symbol blocks, the semi-blind method only performs slightly better than the regular CDMP-trained method and comes within $0.75 \mathrm{~dB}$ of the ideal MMSE equalizer. However, by additionally exploiting knowledge of the unused spreading codes in a practical CDMA system, the semi-blind method outperforms the CDMP-trained method when only a small number of symbol blocks is collected; gains of up to $7 \mathrm{~dB}$ for $I=5$ symbol blocks have been established.

\section{ACKNOWLEDGMENT}

The authors would like to thank S. Zhou of the University of Minnesota, Minneapolisfor his helpful suggestions.

\section{REFERENCES}

[1] T. P. Krauss, W. J. Hillery, and M. D. Zoltowski, "Downlink specific linear equalization for frequency selective CDMA cellular systems," $J$. VLSI Signal Process., vol. 30, no. 3, pp. 143-161, Mar. 2002.

[2] C. D. Frank, E. Visotsky, and U. Madhow, "Adaptive interference suppression for the downlink of a direct sequence CDMA system with long spreading sequences," J. VLSI Signal Process., vol. 30, no. 3, pp. 273-291, Mar. 2002.

[3] F. Petré, G. Leus, L. Deneire, M. Engels, and M. Moonen, "Space-time chip equalizer receivers for WCDMA downlink with code-multiplexed pilot and soft handover," in Proc. GLOBECOM, vol. 1, Nov. 2001, pp. 280-284.

[4] S. Zhou, G. B. Giannakis, and C. Le Martret, "Chip-interleaved blockspread code division multiple access," IEEE Trans. Commun., vol. 50, pp. 235-248, Feb. 2002.

[5] G. Leus, P. Xia, S. Zhou, and G. B. Giannakis, "Chip-interleaved blockspread CDMA or DS-CDMA for cellular downlink?," in Proc. ICASSP, vol. 3, May 2002, pp. 2305-2308.

[6] G. J. Foschini and M. J. Gans, "On limits of wireless communications in a fading environment when using multiple antennas," J. Wireless Person. Commun., vol. 6, no. 3, pp. 311-335, Mar. 1998.

[7] G. G. Raleigh and J. M. Cioffi, "Spatio-temporal coding for wireless communications," IEEE Trans. Commun., vol. 46, pp. 357-366, Mar. 1998.

[8] D. Gesbert, H. Bolcskei, D. Gore, and A. Paulraj, "MIMO wireless channels: Capacity and performance prediction," in Proc. Global Telecommunications Conf. (GLOBECOM), vol. 2, Dec. 2000, pp. 1083-1088.

[9] G. J. Foschini, "Layered space-time architecture for wireless communication in a fading environment when using multiple antennas," Bell Labs Tech. J., vol. 1, no. 2, pp. 41-59, Sept. 1996.

[10] A. Paulraj and T. Kailath, "Increasing Capacity in Wireless Broadcast Systems Using Distributed Transmission/Directional Reception (DTDR)," U.S. Patent 5345 599, Sept. 1994.

[11] V. Tarokh, N. Seshadri, and A. R. Calderbank, "Space-time codes for high data rate wireless communication: Performance criterion and code construction," IEEE Trans. Inform. Theory, vol. 44, pp. 744-765, Mar. 1998.

[12] S. M. Alamouti, "A simple transmit diversity technique for wireless communications," IEEE J. Select. Areas Commun., vol. 16, pp. 1451-1458, Oct. 1998.

[13] V. Tarokh, H. Jafarkhani, and A. R. Calderbank, "Space-time block codes from orthogonal designs," IEEE Trans. Inform. Theory, vol. 45, pp. 1456-1467, July 1999.

[14] E. Lindskog and A. Paulraj, "A transmit diversity scheme for channels with intersymbol interference," in Proc. ICC, vol. 1, June 2000, pp. 307-311.

[15] N. AI-Dhahir, "Single-carrier frequency-domain equalization for space-time block-coded transmissions over frequency-selective fading channels," IEEE Commun. Lett., vol. 5, pp. 304-306, July 2001.

[16] S. Zhou and G. B. Giannakis, "Space-time coding with maximum diversity gains over frequency-selective fading channels," IEEE Signal Processing Lett., vol. 8, pp. 269-272, Oct. 2001.

[17] H. Holma and A. Toskala, WCDMA for UMTS. New York: Wiley, 2001.

[18] A. Stamoulis, Z. Liu, and G. B. Giannakis, "Space-time block-coded OFDMA with linear precoding for multirate services," IEEE Trans. Signal Processing, vol. 50, pp. 119-129, Jan. 2002.

[19] Z. Wang and G. B. Giannakis, "Wireless multicarrier communications: Where Fourier meets Shannon," IEEE Signal Processing Mag., vol. 17, pp. 29-48, May 2000.

[20] L. Deneire, B. Gyselinckx, and M. Engels, "Training sequence versus cyclic prefix, a new look on single-carrier communications," IEEE Commun. Lett., vol. 5, pp. 292-294, July 2001.

[21] G. Leus and M. Moonen, "MUI-free receiver for a synchronous DS-CDMA system based on block spreading in the presence of frequency-selective fading," IEEE Trans. Signal Processing, vol. 48, pp. 3175-3188, Nov. 2000.

[22] Z. Liu, G. B. Giannakis, B. Muquet, and S. Zhou, "Space-time coding for broadband wireless communications," J. Wireless Commun. Mobile Comput., vol. I, no. I, pp. 35-53, Jan.-Mar. 2001.

[23] J. G. Proakis, Digital Communications, 3rd ed. New York: McGrawHill, 1995.

[24] G. H. Golub and C. F. Van Loan, Matrix Computations, 3rd ed. Baltimore, MD: The John Hopkins Univ. Press, 1996.

[25] S. Zhou and G. B. Giannakis, "Single-carrier space-time block coded transmissions over frequency-selective fading channels," IEEE Trans. Inform. Theory, vol. 49, pp. 164-179, Jan. 2003.

[26] A. Klein, G. K. Kaleh, and P. W. Baier, "Zero forcing and minimum mean-square-error equalization for multiuser detection in code-division multiple-access channels," IEEE Trans. Veh. Technol., vol. 14, pp. 1784-1795, Dec. 1996. 
[27] J. G. Proakis, C. M. Rader, F. Ling, C. L. Nikias, M. Moonen, and I. Proudler, Algorithms for Statistical Signal Processing. Englewood Cliffs, NJ: Prentice-Hall, 2002.

[28] E. de Carvalho and D. T. M. Slock, "Deterministic quadratic semi-blind FIR multichannel estimation algorithms and performance," in Proc. ICASSP, vol. 5, June 2000, pp. 2553-2556.

[29] G. Leus and M. Moonen, "Semi-blind channel estimation for block transmissions with nonzero padding," in Proc. Asilomar Conf. Signal, Systems and Computers, vol. 1, Nov. 2001, pp. 762-766.

[30] K. Li and H. Liu, "A new blind receiver for downlink DS-CDMA communications," IEEE Commun. Lett., vol. 3, pp. 193-195, July 1999.

[31] S. Mudulodu and A. Paulraj, "A blind multiuser receiver for the CDMA downlink," in Proc. ICASSP, vol. 5, June 2000, pp. 2933-2936.

[32] D. T. M. Slock and I. Ghauri, "Blind maximum SINR receiver for the DS-CDMA downlink," in Proc. ICASSP, vol. 5, June 2000, pp. 2485-2488.

[33] B. Hochwald, T. L. Marzetta, and C. B. Papadias, "A transmitter diversity scheme for wideband CDMA systems based on space-time spreading," IEEE J. Select. Areas Commun., vol. 19, pp. 48-60, Jan. 2001.

[34] G. Leus, F. Petré, and M. Moonen, "Space-time chip equalization for space-time coded downlink CDMA," in Proc. ICC, vol. 1, Apr. 2002, pp. 568-572.

[35] F. Petré, G. Leus, L. Deneire, M. Moonen, and M. Engels, "Per-tone pilot-trained chip equalizers for space-time coded MC-DS-CDMA downlink," in Proc. ICASSP, vol. 3, May 2002, pp. 2349-2352.

[36] Y. Li, J. C. Chuang, and N. R. Sollenberger, "Transmitter diversity for OFDM systems and its impact on high-rate data wireless networks," IEEE J. Select. Areas Commun., vol. 17, pp. 1233-1243, July 1999.

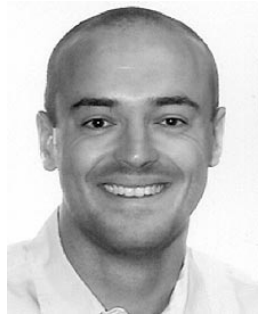

Frederik Petré (M'98-S'99) was born in Tienen, Belgium, in 1974. He received the electrical engineering degree from the Katholieke Universiteit Leuven (KULeuven), Belgium, in 1997. Since 1998, he has been working toward the Ph.D. degree in the Wireless Systems (WISE) Group at Interuniversity Micro-Electronics Center (IMEC), Leuven, Belgium, funded by the Institute for Scientific and Technological Research in Flanders (IWT)

Afterwards, he joined the Interuniversity Micro-Electronics Center (IMEC), Leuven, Belgium, working on wireline transceiver design for twisted pair, cable, and powerline communications. During the fall of 1998, he visited the Information Systems Laboratory (ISL) at Stanford University, Stanford, CA. He is working on the baseband signal processing algorithms and architectures for future wireless communication systems, like third-generation $(3 \mathrm{G})$ and fourth-generation (4G) cellular networks, and wireless local area networks (WLANs). His main research interests are modulation theory, multiple access schemes, channel estimation and equalization, smart antenna and MIMO techniques.

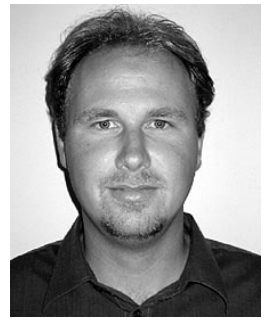

Geert Leus (M'01) was born in Leuven, Belgium, in 1973. He received the electrical engineering degree and the Ph.D. degree in applied sciences from the Katholieke Universiteit Leuven (KULeuven), Belgium, in 1996 and 2000, respectively.

Currently, he is a Postdoctoral Fellow of the Fund for Scientific Research in Flanders (FWO-Vlaanderen) in the Electrical Engineering Department, KULeuven. During the summer of 1998, he visited Stanford University, Stanford, CA, and from March 2001 until May 2002, he was a Visiting Researcher and Lecturer at the University of Minnesota, Minneapolis. His research interests are in the area of signal processing for communications.

Dr. Leus is a Member of the IEEE Signal Processing for Communications Technical Committee, and an Associate Editor for the IEEE TRANSACTIONS ON Wireless COMMUNICATIONS and the IEEE SignAL PROCESSING LeTters.

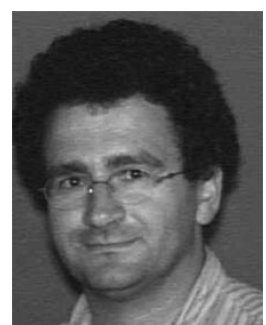

Luc Deneire (S'93-M'99) received the engineering degree in electronics from the University of Liege, Belgium, in 1988, the engineering degree in Telecommunications from the University of Louvain-La-Neuve, Belgium, in 1994, and the Ph.D. in signal processing from Eurecom, Sophia-Antipolis, France, in 1998. During this time, he was a Marie Curie Fellow grant holder.

From 1999 to 2002, he was a Senior Researcher at Interuniversity Micro-Electronics Center (IMEC), Leuven, Belgium, the largest European independent research institute in microelectronics and then joined the University of Nice, Sophia-Antipolis, France, as an Associate Professor. He is working on the signal processing algorithms involved in wireless communications, specifically for third-generation mobile networks, wireless LANs and wireless personal area networks. His main interests are blind and semiblind equalization and channel estimation, modulation theory, multiple access schemes, smart antennas, and link adaptation.

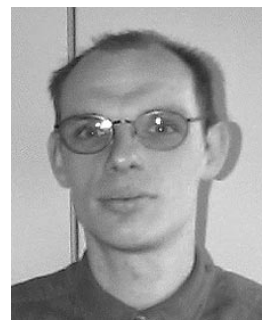

Marc Engels (M'96) received the engineering degree in 1988 and the Ph.D. degree in 1993, both from the Katholieke Universiteit Leuven (KULeuven), Belgium.

He is cofounder of LoraNet, Loranet, Leuven, Belgium, a new company in fixed wireless access, and responsible for research and product development. The company will focus on sub-11-GHz systems that operate under nonline-of-sight conditions. Technologies involved are orthogonal frequency division multiplexing (OFDM) and space-division multiple access (SDMA). He was the Director of the Wireless Department at Interuniversity Micro-Electronics Center (IMEC), Leuven, focused on the implementation of telecommunication systems on a chip. For these systems, he overlooked research on the DSP processing, the mixed-signal RF front-end and the software protocols. He was also active in design methods and tools for implementing multidisciplinary systems. Under his supervision, several systems have been realized, including a $54 \mathrm{Mb} / \mathrm{s}$ WLAN terminal, a GPS-GLONASS receiver, a DECT-GSM dual-mode phone, a cable modem, etc. Previously, he performed research at the Katholieke Universiteit Leuven, Stanford University, Stanford, CA, and the Royal Military School, Brussels, Belgium. He is a Visiting Professor of telecom system design at the KULeuven and of embedded system design at the University of Lugano, Switzerland.

Dr. Engels is an active Member of the KVIV Telecommunications Society and URSI, secretary of the IEEE Benelux Chapter on vehicular technology and telecommunications and Vice-President Member of the Board of Directors of SITEL. He is an Associate Editor for the Wireless Personal Communications Journal and was an Associate Editor of IEEE TRANSACTIONS ON VERY LARGE SCALE INTEGRATION SYSTEMS from 1999 to 2000.

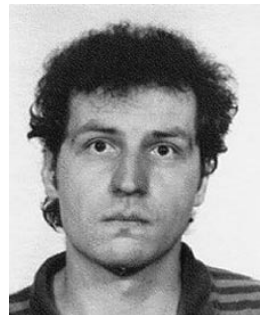

Marc Moonen (M'94) received the electrical engineering degree and the Ph.D. degree in applied sciences from the Katholieke Universiteit Leuven (KULeuven), Belgium, in 1986 and 1990, respectively.

Since 1994, he has been a Research Associate with the Belgian National Fund for Scientific Research (NFWO), and an Associate Professor, since 2000, both in the Electrical Engineering Department, KULeuven. He is Member of the Editorial Board of Integration, the Very Large Scale Integration (VLSI) Journal, Applied Signal Processing. His research activities are in digital signal processing, digital communications, and audio signal processing.

Dr. Moonen received the 1994 KULeuven Research Council Award, the 1997 Alcatel Bell (Belgium) Award (with Piet Vandaele), and was a 1997 Laureate of the Belgium Royal Academy of Science. He was Chairman of the IEEE Benelux Signal Processing Chapter (1998-2002), a EURASIP AdCom Member (European Association for Signal, Speech and Image Processing, 2000), and the IEEE TRANSACTIONS ON CIRCUITS AND SYSTEMS II. 


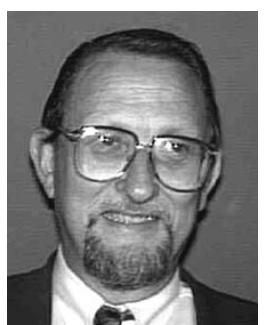

Hugo De Man (M'81-SM'81-F'86) has been a Professor in electrical engineering at the Katholieke Universiteit Leuven (KULeuven), Belgium, since 1976. He was Visiting Associate Professor at University of California, Berkeley, in 1975, teaching semiconductor physics and VLSI design. His early research was devoted to the development of mixed-signal, switched capacitor, and DSP simulation tools, as well as new topologies for high-speed CMOS circuits which lead to the invention of NORA CMOS. In 1984, he was one of the cofounders of the Interuniversity Micro-Electronics Center (IMEC), Leuven, which today, is the largest independent semiconductor research institute in Europe with over 1100 employees. From 1984 to 1995, he was Vice-President of IMEC, responsible for research in design technology for DSP and telecom applications. In 1995, he became a Senior Research Fellow of IMEC, working on strategies for education and research on design of future post-PC systems. His research at IMEC has lead to many novel tools and methods in the area of high level synthesis, hardware-software codesign and $\mathrm{C}++$ based design. Many of these tools are now commercialized by spin-off companies like Coware, Adelante Technologies, and Target Compilers. His work and teaching also resulted in a cluster of DSP oriented companies in Leuven, now known as DSP Valley, where more than 1500 DSP engineers work on design tools and on telecom, networking, and multimedia integrated system products.

Dr. De Man received the Technical Achievement Award of the IEEE Signal Processing Society, the Phil Kaufman Award of the EDA Consortium, and the Golden Jubilee Medal of the IEEE Circuits and Systems Society in 1999. He is a Member of the Royal Academy of Sciences in Belgium. 